Annales Academiæ Scientiarum Fennicæ

Series A. I. Mathematica

Volumen 2, 1976, $41-58$
Commentationes in honorem

Rolf Nevanlinna

LXXX annos nato

\title{
TRANSPORT OF MEASURES AND CONTROL MEASURES
}

\author{
CORNELIU CONSTANTINESCU
}

Let $\Re$ be a $\delta$-ring and let $\mathscr{M}$ be a set of real measures on $\Re$. A control measure for $\mathscr{M}$ is a measure on $\Re$ reflecting some properties of the set $\mathscr{M}$. The properties raised for discussion are the null sets and the regularity of the measures of $\mathscr{M}$. The same problem can be formulated in a more general frame for sets of measures taking their values in different Hausdorff topological commutative groups. In this case it is useless to deal with sets of measures since any such set can be replaced by one measure namely the product of the given measures (taking its values in the product topological group). But while for real valued measures the proper atoms are determined by the null sets, for arbitrary topological groups this is not the case and we have to add the proper atoms to the above two properties defining a control measure. In general the papers dealing with control measures assume that the measure takes its values in a locally convex space. By composing it with the continuous linear forms on this space one gets a set of real valued measures which is easier to handle. If this is not the case the problem becomes by far more complicated. Such a situation appears in [2], where it is assumed that the values of the measure lie in a locally compact commutative group. The main idea consists in transporting the values of a measure from $R / N$ to $R$ by using the fact that the two topological groups are locally isomorphic. The aim of this paper is to generalize the procedure of this paper to more general groups and to replace the local isomorphism with more general mappings (Theorem 2.5). This result is then applied to control measures (Theorem 3.2 and Corollary 3.3).

Throughout this paper we shall denote by $\Re$ a $\delta$-ring (i.e. $\Re \neq \varnothing$ and for any sequence $\left(A_{n}\right)_{n \in N}$ in $\Re$ we have $\cap_{n \in N} A_{n} \in \Re$ and $A_{0} \Delta A_{1} \in \Re$ ) and by $\Re$ a subset of $\Re$ such that the union of any finite family in $\Re$ belongs to $\Re$. We consider $\Re$ ordered by the inclusion relation and denote by $\Lambda_{\Re}=\Lambda$ the set of lower directed subsets of $\mathfrak{R} \backslash\{\varnothing\}$. For any $\mathfrak{A} \in \Lambda$ we denote by $\mathfrak{F}(\mathfrak{U})$ the filter on $\mathfrak{R}$ generated by the filter base 


$$
\{\{B \in \mathfrak{A} \mid B \subset A\} \mid A \in \mathfrak{A}\} \text {. }
$$

If the union of any sequence in $\Re$ belongs to $\Re$ we call $\Re$ a $\sigma$-ring. Let $G$ be a Hausdorff topological commutative group. A G-valued measure on $\Re$ is a map $\mu$ of $\Re$ into $G$ such that for any disjoint sequence $\left(A_{n}\right)_{n \in N}$ in $\Re$ whose union belongs to $\Re$ we have

$$
\mu\left(\cup_{n \in N} A_{n}\right)=\sum_{n \in N} \mu\left(A_{n}\right)
$$

We say that $\mu$ is $\mathfrak{R}$-regular if for any $A \in \Re$ and for any 0-neighbourhood $U$ in $G$ there exists $K \in \mathfrak{\Omega}$ such that $K \subset A$ and

$$
\{\mu(B) \mid B \in \Re, K \subset B \subset A\} \subset \mu(A)+U \text {. }
$$

We say that $\mu$ is exhaustive if for any disjoint sequence $\left(A_{n}\right)_{n \in N}$ in $\Re$ the sequence $\left(\mu\left(A_{n}\right)\right)_{n \in N}$ converges to 0 . We set

$$
\mathfrak{R}(\mu):=\{A \in \mathfrak{R} \mid \forall B \in \mathfrak{\Re}, B \subset A \Rightarrow \mu(B)=0\} .
$$

We say that $\mu$ satisfies ccc if any disjoint family in $\mathfrak{A} \backslash \mathfrak{N}(\mu)$ is countable. Let $\Lambda(\mu)$ be the set of subsets $\mathfrak{A}$ of $\mathfrak{R} \backslash \mathfrak{R}(\mu)$ such that the intersection of any countable family in $\mathfrak{A}$ belongs to $\mathfrak{A}$. The maximal elements of $\Lambda(\mu)$ (for the inclusion relation) will be called atoms of $\mu$. An atom $\mathfrak{A}$ of $\mu$ is called improper if $\mu(\mathfrak{F}(\mathfrak{A}))$ converges to 0 ; otherwise we call $\mathfrak{A}$ proper. A set-atom of $\mu$ is a set $A \in \mathfrak{R} \backslash \mathfrak{N}(\mu)$ such that for any $B \in \mathfrak{R}$ we have $A \cap B \in \mathfrak{N}(\mu)$ or $A \backslash B \in \mathfrak{N}(\mu)$.

\section{Preliminary results}

Propos ition 1.1. Let $\mu$ be a measure on $\Re$ and let $U$ be a neighbourhood of 0 . If $\mu \neq 0$ then there exists $A \in \Re \backslash \mathfrak{R}(\mu)$ such that

$$
\{\mu(B) \mid B \in \Re, B \subset A, A \backslash B \notin \mathfrak{N}(\mu)\} \subset U .
$$

Assume the contrary. Let $A \in \Re \backslash \mathfrak{N}(\mu)$. We construct inductively a sequence $\left(A_{n}\right)_{n \in N}$ in $\Re$ such that we have for any $n \in N$ :

a) $A_{n} \subset A \backslash \underset{m<n}{ } A_{m}$;

b) $\mu\left(A_{n}\right) \notin U$;

c) $A \backslash \underset{m \leqq n}{\bigcup} A_{m} \notin \Re(\mu)$.

Assume that the sequence was constructed up to $n-1$. By c) and by the assumption of the proof there exists $A_{n} \in \Re$ possessing the required properties. The existence of the sequence $\left(A_{n}\right)_{n \in \boldsymbol{N}}$ is a contradiction. 
Proposition 1.2. Let $\mu$ be a measure on $\Re$ and let $U$ be a neighbourhood of 0 such that for any set-atom $D$ of $\mu$ we have $\mu(D) \in U$. Then for any $A \in \mathfrak{R} \backslash \mathfrak{N}(\mu)$ there exists $B \in \mathfrak{R} \backslash \mathfrak{N}(\mu)$ contained in $A$ and such that

$$
\{\mu(C) \mid C \in \Re, C \subset B\} \subset, U .
$$

Let $A \in \Re \backslash \mathfrak{R}(\mu)$. By Proposition 1.1 there exists $D \in \Re \backslash \mathfrak{R}(\mu)$ contained in $A$ such that for any $C \in \Re \backslash \mathfrak{R}(\mu)$ contained in $D$ we have $\mu(D \backslash C) \in U$. If $D$ is a set-atom of $\mu$ then, by the hypothesis, $\mu(D) \in U$ and we get

$$
\{\mu(C) \mid C \in \Re, C \subset D\} \subset U .
$$

In this case we take $B:=D$. If $D$ is not a set-atom of $\mu$ then there exists $B \in \mathfrak{R}$ contained in $D$ such that $B \notin \mathfrak{N}(\mu)$ and $D \backslash B \notin \mathfrak{N}(\mu)$. We get

$$
\{\mu(C) \mid C \in \Re, C \subset B\} \subset U \text {. }
$$

Remark. The above result was proved by K. Musiał ([2], Lemma 1) for measures without set-atoms.

Proposition 1.3. Let $\mathfrak{A}$ be an upper directed subset of $\mathfrak{R}$ and let $\mathfrak{F}$ be the filter on $\mathfrak{R}$ generated by the filter base

$$
\{\{B \in \mathfrak{A} \mid A \subset B\} \mid A \in \mathfrak{A}\} .
$$

Then for any exhaustive measure $\mu$ on $\Re, \mu(\mathfrak{F})$ is a Cauchy filter.

Assume $\mu(\mathfrak{F})$ is not a Cauchy filter. Then there exists a neighbourhood $U$ of 0 such that for any $A \in \mathfrak{U}$ there exists $B \in \mathfrak{A}$ with $A \subset B$ and

$$
\mu(B \backslash A)=\mu(B)-\mu(A) \notin U .
$$

We may construct inductively on increasing sequence $\left(A_{n}\right)_{n \in N}$ in $\mathfrak{A}$ such that $\mu\left(A_{n+1} \backslash A_{n}\right) \notin U$ for any $n \in N$ and this contradicts the hypothesis that $\mu$ is exhaustive.

$\mathrm{Proposition} \mathrm{1.4.} \mathrm{Let} \mu$ be an exhaustive measure on $\Re$ and let $U$ be a neighbourhood of 0 . The set of atoms $\mathfrak{A}$ of $\mu$ for which

is finite.

$$
U \notin \mu(\mathfrak{F}(\mathfrak{U}))
$$

We may assume $U$ closed. Let $\Phi$ be a countable set of atoms of $\mu$ such that

$$
U \notin \mu(\mathfrak{F}(\mathfrak{A}))
$$

for any $\mathfrak{A} \in \Phi$. By [1] Proposition 1.5 there exists a disjoint family $\left(A_{\mathfrak{A}}\right)_{\mathfrak{Q} \in \Phi}$ in $\mathfrak{R}$ such that $A_{\mathfrak{U}} \in \mathfrak{U}$ for any $\mathfrak{U} \in \Phi$. For any $\mathfrak{U} \in \Phi$ there exists $B_{\mathfrak{U}} \in \mathfrak{A}$ such that $B_{\mathfrak{U}} \subset A_{\mathfrak{U}}$ and $\mu\left(B_{\mathfrak{Y}}\right) \notin U$. Since $\mu$ is exhaustive and since $\left(B_{\mathfrak{U}}\right)_{\mathfrak{Q} \in \Phi}$ is a disjoint family in $\mathfrak{R}$ it follows that $\Phi$ is finite. 
Proposition 1.5. If the one point sets of a topological commutative group $G$ are $G_{\delta}$-sets then any exhaustive $G$-valued measure on $\Re$ satisfies ccc.

Let $\mu$ be an exhaustive $G$-valued measure on $\Re$ and let $\left(U_{n}\right)_{n \in N}$ be a sequence of 0 -neighbourhoods in $G$ whose intersection is $\{0\}$. Let further $\left(A_{\iota}\right)_{\iota \in I}$ be a family in $\Re \backslash \Re(\mu)$ and let $\left(B_{\iota}\right)_{\iota \in I}$ be a family in $\Re$ such that $\mu\left(B_{\iota}\right) \neq 0$ and $B_{\iota} \subset A_{\imath}$ for any $\iota \in I$. Since $\mu$ is exhaustive the set

is finite. From

$$
\left\{\iota \in I \mid \mu\left(B_{\iota}\right) \notin U_{n}\right\}
$$

$$
I=\bigcup_{n \in N}\left\{\iota \in I \mid \mu\left(B_{\imath}\right) \notin U_{n}\right\}
$$

it follows that $I$ is countable.

Proposition 1.6. Let $\mu$ be a measure on $\Re$ and let $\mathfrak{F}$ be the filter on $\Re$ generated by the filter base

$$
\{B \in \Re \mid B \cap A=\varnothing\} \mid A \in \Re\} .
$$

The following assertions are equivalent:

a) $\mu$ is exhaustive;

b) for any disjoint sequence $\left(A_{n}\right)_{n \in N}$ in $\Re$ and for any neighbourhood $U$ of 0 there exists $m \in N$ such that

for any $n \geqq m$;

$$
\left\{\mu(A) \mid A \in \Re, A \subset A_{n}\right\} \subset U
$$

c) $\mu(\mathfrak{F})$ converges to 0 .

$\mathrm{a} \Rightarrow \mathrm{b}$. If $\mathrm{b}$ ) does not hold there exists a strictly increasing sequence $\left(k_{n}\right)_{n \in N}$ in $N$ and for each $n \in N$ a $B_{n} \in \Re$ with $B_{n} \subset A_{k_{n}}$ and $\mu\left(B_{n}\right) \notin U$. Since $\left(B_{n}\right)_{n \in N}$ is a disjoint sequence in $\Re \mu$ is not exhaustive.

$\mathrm{b} \Rightarrow \mathrm{c}$. Assume $\mu(\mathfrak{F})$ does not converge to 0 . Then there exists a neighbourhood $U$ of 0 such that for any $A \in \Re$ there exists $B \in \Re$ such that $B \cap A=\varnothing$ and $\mu(B) \notin U$. We may construct inductively a disjoint sequence $\left(A_{n}\right)_{n \in N}$ in $\Re$ such that $\mu\left(A_{n}\right) \notin U$ for any $n \in N$ and this contradicts b).

$\mathrm{c} \Rightarrow \mathrm{a}$. Let $\left(A_{n}\right)_{n \in N}$ be a disjoint sequence in $\Re$. Let $U$ be an arbitrary neighbourhood of 0 and let $V$ be a neighbourhood of 0 such that $V+V \subset U$. There exists $A \in \Re$ such that

Since

$$
\{\mu(B) \mid B \in \Re, B \cap A=\varnothing\} \subset V .
$$

$$
\mu\left(\cup\left(A \cap A_{n}\right)\right)=\sum_{n \in N} \mu\left(A \cap A_{n}\right)
$$

there exists $m \in N$ such that $\mu\left(A \cap A_{n}\right) \in V$ for any $n \geqq m$. We get

$$
\mu\left(A_{n}\right)=\mu\left(A \cap A_{n}\right)+\mu\left(A_{n} \backslash A\right) \in V+V \subset U
$$

for any $n \geqq m$. Hence $\mu$ is exhaustive. 


\section{Transport of measures}

Throughout this section we shall denote by $G, H$ Hausdorff topological commutative groups, by $p$ a continuous semi-value on $G$ (i.e. a map of $G$ into $\boldsymbol{R}_{+}$such that

a) $p(0)=0$;

b) $p(x+y) \leqq p(x)+p(y)$;

c) $p(-x)=p(x)$

for any $x, y \in G)$, and by $U$ the set $\{x \in G \mid p(x) \leqq 1\}$.

Proposition 2.1. Let $\mu$ be an exhaustive G-valued measure on $\Re$ such that

$$
\lim p(\mu(\mathfrak{F}(\mathfrak{A}))) \leqq 1
$$

for any atom $\mathfrak{A}$ of $\mu$. Then there exists a countable subset $\mathfrak{B}$ of $\mathfrak{R}$ such that:

a) for any $B \in \mathfrak{B}$ we have

$$
\{\mu(A) \mid A \in \Re, A \subset B\} \subset U \text {; }
$$

b) for any $A \in \Re$ with $A \cap\left(\cup_{B \in \mathfrak{B}} B\right)=\varnothing$ we have $p(\mu(A))=0$.

If $\mathfrak{R}$ is a $\sigma$-ring we may even assume $\mathfrak{B}$ finite.

From the properties of $p$ it follows that $p^{-1}(0)$ is a closed subgroup of $G$ and $G / p^{-1}(0)$ is a Hausdorff topological commutative group. Let $u$ be the canonical map $G \rightarrow G / p^{-1}(0)$. Then $u \circ \mu$ is an exhaustive measure on $\Re$. Let $A$ be a set-atom of $u \circ \mu$ and let

$$
\mathfrak{U}_{A}:=\{B \in \mathfrak{R} \mid A \backslash B \in \mathfrak{R}(u \circ \mu)\} .
$$

By [1] Proposition 1.2 b) $\mathfrak{H}_{A}$ is an atom of $u \circ \mu$. Since $\mathfrak{R}(\mu) \subset \mathfrak{R}(u \circ \mu)$ it follows that $\mathfrak{Y}_{A}$ is an atom of $\mu$ ([1], Corollary 1.4). We get

$$
u \circ \mu(A)=\lim u \circ \mu\left(\mathfrak{F}\left(\mathfrak{H}_{A}\right)\right) \in u(U) .
$$

We may assume $u \circ \mu \neq 0$. Let $\mathfrak{B}^{\prime}$ be the set of $B \in \mathfrak{R} \backslash \mathfrak{R}(u \circ \mu)$ such that

$$
\{\mu(A) \mid A \in \Re, A \subset B\} \subset U
$$

and let $\Omega$ be the set of subsets $\mathfrak{B}^{\prime \prime}$ of $\mathfrak{B}^{\prime}$ such that the sets of $\mathfrak{B}^{\prime \prime}$ are pairwise disjoint. By Zorn's theorem there exists a maximal element $\mathfrak{B}$ of $\Omega$ with respect to the inclusion relation. By Proposition $1.5 u \circ \mu$ satisfies ccc. Hence $\mathfrak{B}$ is countable.

Let $A \in \Re$ with $A \cap\left(\cup_{B \in \mathfrak{B}} B\right)=\varnothing$ and $p(\mu(A)) \neq 0$. By the first part of the proof and Proposition 1.2 there exists $B \in \mathfrak{B}^{\prime}$ contained in $A$. Then $\mathfrak{B} \cup\{B\} \in \Omega$ and this contradicts the maximality of $\mathfrak{B}$. Hence $\mathfrak{B}$ fulfills $\mathrm{b})$. 
Assume now that $\Re$ is a $\sigma$-ring of sets and let $\left(B_{n}\right)_{n \in N}$ be a disjoint sequence in $\Re$ such that

$$
\mathfrak{B}:=\left\{B_{n} \mid n \in N\right\} .
$$

Since $\left(\cup_{m \geqq n} B_{m}\right)_{n \in \mathbf{N}}$ is a decreasing sequence in $\Re$ whose intersection is empty there exists $n \in N$ such that $\bigcup_{m \geqq n} B_{m} \in \mathfrak{B}^{\prime}$ and we may replace $\mathfrak{B}$ by

$$
\left\{B_{m} \mid m<n\right\} \cup\left\{\underset{m \geqq n}{\cup} B_{m}\right\} \text {. }
$$

Remark. This result was proved by K. Musial ([2], Proposition 2) for atomless measures defined on $\sigma$-rings and satisfying ccc.

Corollary 2.2. Let $G_{0}$ be an open subgroup of $G$ and let $\mu$ be a G-valued measure on $\Re$ such that for any atom $\mathfrak{A}$ of $\mu$ there exists $A \in \mathfrak{A}$ with

Then $\mu(\Re) \subset G_{0}$.

$$
\{\mu(B) \mid B \in \mathfrak{A}, B \subset A\} \subset G_{0} .
$$

Since $G_{0}$ is open and closed we may find a $p$ such that $G_{0}=U$. Let $A \in \Re$. By the proposition there exists a finite partition $\left(A_{\iota}\right)_{t \in I}$ of $A$ with sets of $\Re$ such that $\mu\left(A_{\iota}\right) \in G_{0}$ for any $\iota \in I$. We get $\mu(A) \in G_{0}$.

Remark. This result was proved by K. Musiał ([2], Proposition 3) for atomless measures defined on $\sigma$-rings and satisfying cce.

Let $M$ be a subset of $G$. A map $u$ of $M$ into $H$ is called an $\omega$-map if it possesses the following property: Let $\Re^{\prime}$ be a $\sigma$-ring, let $\mathfrak{A}$ be an upper directed subset of $\Re^{\prime}$, let $\mathfrak{F}$ be the filter on $\mathfrak{R}^{\prime}$ generated by the filter base

$$
\{\{B \in \mathfrak{A} \mid A \subset B\} \mid A \in \mathfrak{A}\},
$$

and let $\mu$ be a $G$-valued measure on $\Re^{\prime}$ such that $\mu\left(\Re^{\prime}\right) \subset M$ and such that $\mu(\mathfrak{F})$ converges to a point of $M$. Then the map

$$
A \mapsto u(\mu(A)): \Re^{\prime} \rightarrow H
$$

is a measure and the image of $\mathfrak{F}$ through it converges to $u(\lim \mu(\mathfrak{F}))$. It follows immediately that if $\mathfrak{A} \in \Lambda$ and if $\mu$ is a $K$-regular $G$-valued measure on $\mathfrak{R}$ such that $\mu(\mathfrak{R}) \subset M$ and such that $\mu(\mathfrak{F}(\mathfrak{A}))$ converges to a point of $M$ then the map

$$
A \mapsto u(\mu(A)): \Re \rightarrow H
$$

is a $\mathfrak{R}$-regular measure and the image of $\mathfrak{F}(\mathfrak{U})$ through it converges to $u(\lim \mu(\mathfrak{F}(\mathfrak{A})))$. It is obvious that if $u$ is an $\omega$-map of $M$ into $H$ then

$$
u(x+y)=u(x)+u(y)
$$

for any $x, y \in M$ with $x+y \in M$. This condition together with the continuity assures that $u$ is an $\omega$-map, but the continuity is not necessary. 
Proposition 2.3. Let $\mu$ be a $\mathfrak{R}$-regular G-valued measure on $\Re$ such that

$$
\lim p(\mu(\mathfrak{F}(\mathfrak{A}))) \leqq 1
$$

for any atom $\mathfrak{A}$ of $\mu$ and let $u$ be an $\omega$-map of $U$ into $H$. Then there exists a unique $H$-valued measure $v$ on $\Re$ such that

$$
v(A)=u(\mu(A))
$$

for any $A \in \Re$ with

$$
\{\mu(B) \mid B \in \Re, B \subset A\} \subset U .
$$

For any $A \in \Re$ with $\mu(A) \in U$ and $u(\mu(A)) \neq 0$ we have $A \notin \mathfrak{R}(v)$. $v$ is $\mathfrak{R}$-regular. If $u(x)=0$ implies $x=0$ then $\mathfrak{N}(\mu)=\mathfrak{N}(v)$.

Let $\mathfrak{A}$ be the set of $A \in \Re$ such that

$$
\{\mu(B) \mid B \in \Re, B \subset A\} \subset U .
$$

Then the map

$$
B \mapsto u(\mu(B)):\{B \in \mathfrak{A} \mid B \subset A\} \rightarrow H
$$

is a $\{K \in \mathfrak{H} \mid K \subset A\}$-regular measure for any $A \in \mathfrak{A}$. Let $\Omega$ be the set of finite disjoint subsets of $\mathfrak{A}$. Let $\mathfrak{B}^{\prime}, \mathfrak{B}^{\prime \prime} \in \Omega$ such that

$$
\underset{B^{\prime} \in \mathfrak{B}^{\prime}}{\cup} B^{\prime}=\underset{B^{\prime \prime} \in \mathfrak{B}^{\prime \prime}}{\cup} B^{\prime \prime}
$$

Then $\mu\left(B^{\prime} \cap B^{\prime \prime}\right) \in U$ for any $\left(B^{\prime}, B^{\prime \prime}\right) \in \mathfrak{B}^{\prime} \times \mathfrak{B}^{\prime \prime}$ and we get

$$
\begin{aligned}
& \sum_{B^{\prime} \in \mathfrak{B}^{\prime}} u\left(\mu\left(B^{\prime}\right)\right)=\sum_{B^{\prime} \in \mathfrak{B}^{\prime}} \sum_{B^{\prime \prime} \in \mathfrak{B}^{\prime \prime}} u\left(\mu\left(B^{\prime} \cap B^{\prime \prime}\right)\right) \\
= & \sum_{B^{\prime \prime} \in \mathfrak{B}^{\prime \prime}} \sum_{B^{\prime} \in \mathfrak{B}^{\prime}} u\left(\mu\left(B^{\prime} \cap B^{\prime \prime}\right)\right)=\sum_{B^{\prime \prime} \in \mathfrak{B}^{\prime \prime}} u\left(\mu\left(B^{\prime \prime}\right)\right) .
\end{aligned}
$$

Let $A \in \Re$. By Proposition 2.1 there exists $\mathfrak{B} \in \Omega$ such that $A=\mathrm{U}_{B \in \mathfrak{B}} B$. By the above considerations the element of $H$

$$
\sum_{B \in \mathfrak{B}} u(\mu(B))
$$

depends only on $A$. We shall denote it by $v(A)$. It is obvious that $\nu(A)=u(\mu(A))$ for any $A \in \mathfrak{A}$. Let $\left(A_{\iota}\right)_{\iota \in I}$ be a countable disjoint family in $\Re$ whose union belongs to $\mathfrak{R}$ and let $\mathfrak{B} \in \Omega$ such that

Then

$$
\underset{B \in \mathfrak{B}}{\cup} B=\bigcup_{\iota \in I} A_{\imath} .
$$




$$
\begin{gathered}
v\left(\bigcup_{\imath \in I} A_{\imath}\right)=\sum_{B \in \mathfrak{B}} u(\mu(B))=\sum_{B \in \mathfrak{B}} \sum_{\imath \in I} u\left(\mu\left(B \cap A_{\imath}\right)\right) \\
=\sum_{\imath \in I} \sum_{B \in \mathfrak{\Re}} u\left(\mu\left(B \cap A_{\imath}\right)\right)=\sum_{\imath \in I} v\left(A_{\imath}\right) .
\end{gathered}
$$

Hence $v$ is a measure.

Let $\lambda$ be an $H$-valued measure on $\Re$ such that

$$
\lambda(A)=u(\mu(A))
$$

for any $A \in \mathfrak{A}$. Let $A \in \mathfrak{R}$ and let $\mathfrak{B} \in \Omega$ such that

$$
A=\underset{B \in \mathfrak{B}}{\cup} B
$$

We get

$$
\lambda(A)=\sum_{B \in \mathfrak{B}} \lambda(B)=\sum_{B \in \mathfrak{B}} u(\mu(B))=\sum_{B \in \mathfrak{B}} v(B)=v(A) .
$$

This proves the unicity of $v$.

Let $A \in \mathfrak{R}(v)$ with $\mu(A) \in U$ and let $\mathfrak{B} \in \Omega$ such that

$$
A=\underset{B \in \mathfrak{B}}{\cup} B
$$

Then

for any $B \in \mathfrak{B}$. Hence

$$
u(\mu(B))=v(B)=0
$$

$$
u(\mu(A))=0 \text {. }
$$

In order to show that $v$ is $\Re$-regular let $A \in \Re$ and let $V$ be a 0 neighbourhood in $H$. Let $\mathfrak{B} \in \Omega$ such that

$$
A=\underset{B \in \mathfrak{B}}{\cup} B
$$

let $n$ be the cardinal of $\mathfrak{B}$, and let $W$ be a 0 -neighbourhood in $H$ such that $n W \subset V$. For any $B \in \mathfrak{B}$ there exists $K_{B} \in \mathfrak{R}$ contained in $B$ such that

$$
v(C) \in v(B)+W
$$

for any $C \in \Re$ with $K_{B} \subset C \subset B$. We set

$$
K:=\underset{B \in \mathfrak{B}}{\cup} K_{B} \in \mathfrak{R} \text {. }
$$

Let $C \in \Re$ with $K \subset C \subset A$. Then

$$
v(C)=\sum_{B \in \mathfrak{B}} v(C \cap B) \in \sum_{B \in \mathfrak{B}}(v(B)+W) \subset v(A)+V .
$$

Hence $v$ is $\Re$-regular. 
It is obvious that $\mathfrak{R}(\mu) \subset \mathfrak{R}(v)$. Assume $u(x)=0$ implies $x=0$ and let $A \in \mathfrak{N}(v)$. Let $\mathfrak{B} \in \Omega$ such that

$$
A=\underset{B \in \mathfrak{B}}{\cup} B
$$

Let $C \in \mathfrak{R}, C \subset A$. Then for any $B \in \mathfrak{B}$ we have

$$
u(\mu(B \cap C))=v(B \cap C)=0
$$

and therefore $\mu(B \cap C)=0$. We get $\mu(C)=0$. Hence $A \in \mathfrak{N}(\mu)$.

Remark. This Proposition is a generalization of a theorem of Herer ([2], Theorem 4).

Proposition 2.4. Let $\Phi$ be a set of maximal elements of $\Lambda$ such that: a) we have $\cap_{\mathfrak{A} \in \Psi} \mathfrak{A}=\varnothing$ for any infinite subset $\Psi$ of $\Phi$; b) for any $\mathfrak{A} \in \Phi$ and for any $A \in \mathfrak{U}$ there exists $K \in \mathfrak{A} \cap \mathfrak{\Re}$ with $K \subset A$; c) for any $\mathfrak{A} \in \Phi$ the intersection of any countable family in $\mathfrak{A}$ belongs to $\mathfrak{A}$. Let further $\left(A_{\mathfrak{Q}}\right)_{\mathfrak{Q} \in \Phi}$ be a family in $\mathfrak{i}$ such that $A_{\mathfrak{Q}} \in \mathfrak{U} \backslash \mathfrak{\mathfrak { U } ^ { \prime }}$ for any $\mathfrak{A}$, $\mathfrak{H}^{\prime} \in \Phi, \mathfrak{A} \neq \mathfrak{A}^{\prime}$, let $\left(x_{\mathfrak{Q}}\right)_{\mathfrak{A} \in \Phi}$ be a family in $G$, and let $\mu$ be a $\mathfrak{A} \backslash \cup_{\mathfrak{A} \in \Phi} \mathfrak{H}-$ regular $G$-valued measure on $\mathfrak{R} \backslash \cup_{\mathfrak{Q} \in \Phi} \mathfrak{H}^{1}$. There exists a unique $G$-valued measure $v$ on $\mathfrak{i}$ equal to $\mu$ on $\mathfrak{R} \backslash \cup_{\mathfrak{A} \in \Phi} \mathfrak{A}$ and equal to $x_{\mathfrak{A}}$ at $A_{\mathfrak{A}}$ for any $\mathfrak{A} \in \Phi . \nu$ is $\mathfrak{R}$-regular.

Let $\leqq$ be a linear order relation on $\Phi$. For any $\left(A, \mathfrak{A}_{0}\right) \in \Re \times \Phi$ we set:

$$
\begin{aligned}
& \Phi(A):=\{\mathfrak{A} \in \Phi \mid A \in \mathfrak{A}\}, \\
& \Phi\left(A, \mathfrak{A}_{0}\right):=\left\{\mathfrak{A} \in \Phi(A) \mid \mathfrak{A}<\mathfrak{A}_{0}\right\}, \\
& \nu(A):=\mu\left(A \backslash \underset{\mathfrak{U} \in \Phi(A)}{\cup} A_{\mathfrak{U}}\right)+\sum_{\mathfrak{U} \in \Phi(A)}\left(x_{\mathfrak{Q}}-\mu\left(A_{\mathfrak{Q}} \backslash\left(A \backslash \underset{\mathfrak{U}^{\prime} \in \Phi(A, \mathfrak{A})}{\cup} A_{\mathfrak{Q} \mathfrak{Y}^{\prime}}\right)\right)\right) ;
\end{aligned}
$$

by a) $\Phi(A)$ is finite and therefore $\nu(A)$ is well defined. Let $\left(B_{\iota}\right)_{\iota \in I}$ be a countable disjoint family in $\Re$ whose union $B$ belongs to $\Re$. Since the elements of $\Phi$ are maximal elements of $\Lambda$ we deduce by c) that $\left(\Phi\left(B_{b}\right)\right)_{\iota \in I}$ is a disjoint family whose union is $\Phi(B)$. We have

$$
\begin{aligned}
& \nu(B)=\mu\left(B \backslash \underset{\mathfrak{A} \in \Phi(B)}{\cup} A_{\mathfrak{Y}}\right)+\sum_{\mathfrak{U} \in \Phi(B)}\left(x_{\mathfrak{Q}}-\mu\left(A_{\mathfrak{U}} \backslash\left(B \backslash \underset{\mathfrak{U}^{\prime} \in \Phi(B, \mathfrak{U})}{\cup} A_{\mathfrak{Q} \mathfrak{U}^{\prime}}\right)\right)\right)
\end{aligned}
$$

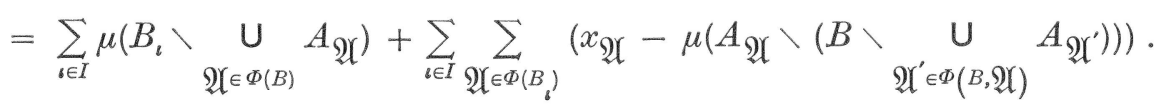

Let $\iota \in I$. Then

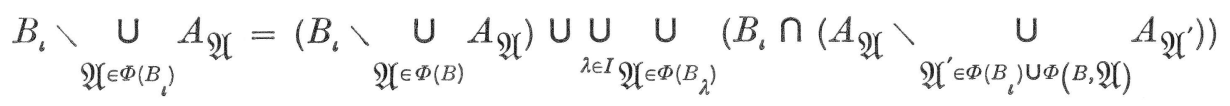

\footnotetext{
${ }^{1}$ By [1], Proposition 1.3, $\mathfrak{R} \backslash \bigcup_{\mathfrak{A} \in \Phi} \mathfrak{A}$ is a $\delta$-ring.
} 
and therefore

$$
\begin{aligned}
& \mu\left(B_{\imath} \backslash \underset{\mathfrak{A} \in \mathbb{\Phi}\left(B_{\iota}\right)}{\cup} A_{\mathfrak{Q}}\right)
\end{aligned}
$$

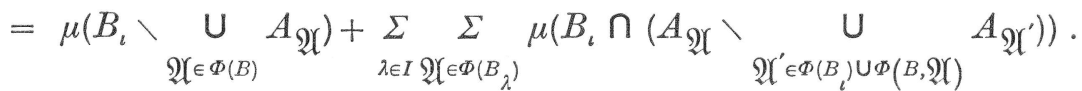

We have for any $\mathfrak{A} \in \Phi\left(B_{\iota}\right)$

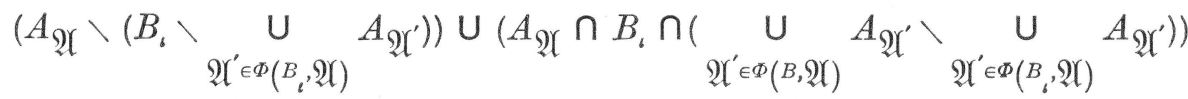

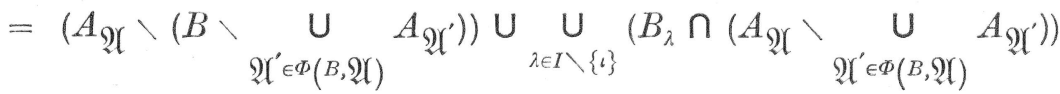

and therefore

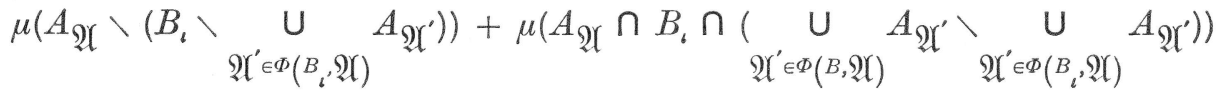

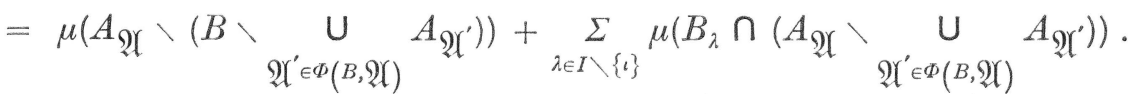

We get

$$
\begin{aligned}
& \nu(B)=\sum_{\imath \in I} \mu\left(B_{\iota} \backslash \underset{\mathfrak{A} \in \Phi\left(B_{\iota}\right)}{\cup} A_{\mathfrak{A}}\right) \\
& -\sum_{\imath \in I} \sum_{\lambda \in I} \sum_{\mathfrak{U} \in \Phi\left(B_{\lambda}\right)} \mu\left(B_{\imath} \cap\left(A_{\mathfrak{U}} \backslash \underset{\mathfrak{U}^{\prime} \in \Phi(B) \cup \Phi(B, \mathfrak{U})}{\cup} A_{\left.\mathfrak{U} \mathfrak{U}^{\prime}\right)}\right)\right. \\
& +\sum_{i \in I} \sum_{\mathfrak{U} \in \Phi\left(B_{\imath}\right)}\left(x_{\mathfrak{Q}}-\mu\left(A_{\mathfrak{Q}} \backslash\left(B_{\iota} \backslash \underset{\mathfrak{U}^{\prime} \in \Phi\left(B_{\imath}, \mathfrak{U}\right)}{\cup} A_{\mathfrak{Q} \mathfrak{U}^{\prime}}\right)\right)\right)
\end{aligned}
$$

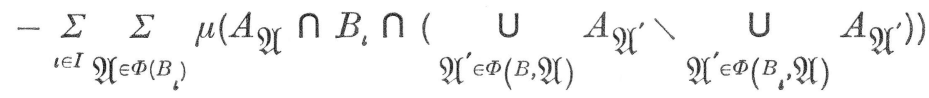

$$
\begin{aligned}
& +\sum_{\imath \in I} \sum_{\mathfrak{U} \in \Phi\left(B_{\mathfrak{\imath}}\right)} \sum_{\lambda \in I \backslash\{\mathfrak{l}\}} \mu\left(B_{\lambda} \cap\left(A_{\mathfrak{Q} \mathfrak{Y}} \backslash \underset{\mathfrak{U}^{\prime} \in \Phi(B, \mathfrak{A})}{\mathrm{U}} A_{\mathfrak{H}^{\prime}}\right)\right) \\
& =\sum_{\imath \in I} v\left(B_{\iota}\right)-\sum_{\iota \in I}\left(\sum_{\lambda \in I} \underset{\mathfrak{A} \in \Phi\left(B_{\lambda}\right)}{\sum} \mu\left(B_{\iota} \cap\left(A_{\mathfrak{U}} \backslash \underset{\mathfrak{U}^{\prime} \in \Phi\left(B_{\iota}\right) \cup \Phi(B, \mathfrak{U})}{\cup} A_{\mathfrak{U} \mathfrak{U}^{\prime}}\right)\right)\right. \\
& +\underset{\mathfrak{U} \in \Phi\left(B_{\iota}\right)}{\sum_{\iota}} \mu\left(B_{\iota} \cap A_{\mathfrak{Q}} \cap\left(\underset{\mathfrak{U}^{\prime} \in \Phi(B, \mathfrak{U})}{\cup} A_{\mathfrak{Y}^{\prime}} \backslash \underset{\mathfrak{U}^{\prime} \in \Phi(B, \mathfrak{U})}{\mathrm{U}} A_{\mathfrak{Y}^{\prime}}\right)\right) \\
& -\sum_{\lambda \in I \backslash\{\mathfrak{}\}} \sum_{\mathfrak{U} \in \Phi\left(B_{\lambda}\right)} \mu\left(B_{\imath} \cap\left(A_{\mathfrak{U}} \backslash \underset{\mathfrak{U}^{\prime} \in \Phi(B, \mathfrak{U})}{\cup} A_{\mathfrak{U}^{\prime}}\right)\right) .
\end{aligned}
$$


We have for any $\iota \in I$

$$
\begin{aligned}
& \underset{\mathfrak{A} \in \Phi\left(B \backslash B_{\iota}\right)}{\mathrm{U}} B_{\iota} \cap\left(A_{\mathfrak{Y}} \backslash \underset{\mathfrak{A}^{\prime} \in \Phi(B, \mathfrak{A})}{\mathrm{U}} A_{\mathfrak{U}^{\prime}}\right) \\
& =\left(\underset{\mathfrak{U} \in \Phi(B)}{\cup} B_{\imath} \cap\left(A_{\mathfrak{U}} \backslash \underset{\mathfrak{U}^{\prime} \in \Phi(B) \cup \Phi(B, \mathfrak{U})}{\mathrm{U}} A_{\mathfrak{Y}^{\prime}}\right)\right) \\
& \mathrm{U}\left(\underset{\mathfrak{A} \in \Phi\left(B_{\iota}\right)}{\cup} B_{\imath} \cap A_{\mathfrak{A}} \cap\left(\underset{\mathfrak{U}^{\prime} \in \Phi(B, \mathfrak{U})}{\mathrm{U}} A_{\mathfrak{U}^{\prime}} \backslash \underset{\mathfrak{U}^{\prime} \in \Phi\left(B_{\iota}, \mathfrak{U}\right)}{\cup} A_{\left.\mathfrak{U}^{\prime}\right)}\right),\right.
\end{aligned}
$$

and therefore

$$
\begin{aligned}
& \sum_{\lambda \in I \backslash\{b\}} \sum_{\mathfrak{U} \in \Phi\left(B_{\lambda}\right)} \mu\left(B_{\imath} \cap\left(A_{\mathfrak{Q}} \backslash \underset{\mathfrak{U}^{\prime} \in \Phi(B, \mathfrak{A})}{\cup} A_{\mathfrak{U}^{\prime}}\right)\right) \\
& =\sum_{\lambda \in \mathbb{I}} \underset{\mathfrak{A} \in \Phi\left(B_{\lambda}\right)}{\Sigma} \mu\left(B_{\imath} \cap\left(A_{\mathfrak{Q}} \backslash \underset{\mathfrak{U}^{\prime} \in \Phi\left(B_{\iota}\right) \cup \Phi(B, \mathfrak{Q})}{U} A_{\mathfrak{U}^{\prime}}\right)\right)
\end{aligned}
$$

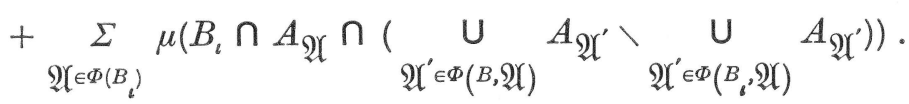

We get

$$
v(B)=\sum_{\iota \in I} v\left(B_{\iota}\right)
$$

i.e. $v$ is a measure. It is obvious that the restriction of $v$ to $\Re \backslash \cup_{\mathfrak{A} \in \Phi} \mathfrak{A}$ is equal to $\mu$ and that $\nu\left(A_{\mathfrak{Q}}\right)=x_{\mathfrak{A}}$ for any $\mathfrak{A} \in \Phi$. Let $\lambda$ be a $G$-valued measure on $\Re$ possessing these two last properties of $v$ and let $A \in \Re$. Then

and therefore

$$
A=\left(A \backslash \underset{\mathfrak{H} \in \Phi(A)}{\cup} A_{\mathfrak{Q} \mathfrak{U}}\right) \cup \underset{\mathfrak{A} \in \Phi(A)}{\cup}\left(A \cap\left(A_{\mathfrak{U}} \backslash \underset{\mathfrak{U}^{\prime} \in \Phi(A, \mathfrak{Q})}{\cup} A_{\mathfrak{A}^{\prime}}\right)\right)
$$

$$
\lambda(A)=\mu\left(A \backslash \underset{\mathfrak{Q} \in \Phi(A)}{\cup} A_{\mathfrak{Q}}\right)+\underset{\mathfrak{A} \in \Phi(A)}{\Sigma} \lambda\left(A \cap\left(A_{\mathfrak{Q}} \backslash \underset{\mathfrak{Q} \mathfrak{Y}^{\prime} \in \Phi(A, \mathfrak{U})}{\cup} A_{\mathfrak{Q} \mathfrak{U}^{\prime}}\right)\right)
$$

We have for any $\mathfrak{A} \in \Phi(A)$

and therefore

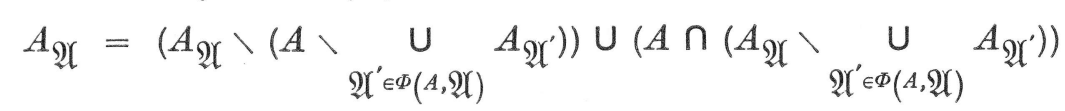

$$
x_{\mathfrak{U}}=\mu\left(A_{\mathfrak{U}} \backslash\left(A \backslash \underset{\mathfrak{U}^{\prime} \in \Phi(A, \mathfrak{U})}{\cup} A_{\left.\mathfrak{U}^{\prime}\right)}\right)+\lambda\left(A \cap\left(A_{\mathfrak{Q}} \backslash \underset{\mathfrak{U}^{\prime} \in \Phi\left(A, \mathfrak{U}^{\prime}\right)}{\mathrm{U}} A_{\mathfrak{U}^{\prime}}\right)\right)\right.
$$

and we get

$$
\begin{aligned}
\lambda(A)= & \mu\left(A \backslash \underset{\mathfrak{A} \in \Phi(A)}{\cup} A_{\mathfrak{A}}\right) \\
& +\sum_{\mathfrak{A} \in \Phi(A)}\left(x_{\mathfrak{Q}}-\mu\left(A_{\mathfrak{Q}} \backslash\left(A \backslash \underset{\mathfrak{A}^{\prime} \in \Phi(A, \mathfrak{A})}{\mathrm{U}} A_{\mathfrak{U}^{\prime}}\right)\right)=v(A) .\right.
\end{aligned}
$$


This proves the uniqueness of $v$.

Let $A \in \Re$ and let $V$ be a 0 -neighbourhood in $G$. By b) there exists for any $\mathfrak{A} \in \Phi(A)$ a $K_{\mathfrak{Q}} \in \mathfrak{N} \cap \mathfrak{A}$ contained in $A$. Since $\mu$ is $\mathfrak{N} \backslash \cup_{\mathfrak{A} \in \Phi} \mathfrak{A}$-regular there exists a $K \in \mathfrak{K}$ contained in $A \backslash \cup_{\mathfrak{A} \in \Phi(A)} K_{\mathfrak{A}}$ such that $\mu(B) \in V$ for any $B \in \mathfrak{X} \backslash \cup_{\mathfrak{Q} \in \Phi} \mathfrak{A}$ contained in

$$
\left(A \backslash \underset{\mathfrak{U} \in \Phi(A)}{\cup} K_{\mathfrak{A}}\right) \backslash K \text {. }
$$

We get $v(B) \in V$ for any $B \in \mathfrak{R}$ contained in $A \backslash\left(K \cup \cup_{\mathfrak{A} \in \Phi(A)} K_{\mathfrak{Q}}\right)$. This shows that $v$ is $\mathfrak{\AA}$-regular.

Th e o r e m 2.5. Let $\mu$ be a $\mathfrak{\AA}$-regular $G$-valued measure on $\Re$ and let $u$ be an $\omega$-map of $U$ into $H$. Then there exists a $\Re$-regular $H$-valued measure $v$ on $\Re$ such that: a) $\nu(A)=u(\mu(A))$ for any $A \in \Re$ with

$$
\{\mu(B) \mid B \in \Re, B \subset A\} \subset U \text {; }
$$

b) any proper atom of $v$ is a proper atom of $\mu$. If $H \neq\{0\}$ then for any 0-neighbourhood $V$ in $G$ there exists a $v$ with the above properties and such that: c) any atom $\mathfrak{A}$ of $\mu$ is a proper atom of $v$ if for any $A \in \mathfrak{A}$ there exists $B \in \mathfrak{H}$ with $B \subset A$ and $\mu(B) \notin V$. If moreover $u(x)=0$ implies $x=0$ we may choose $v$ such that besides the above properties the following ones hold: d) $\mathfrak{N}(\mu)=\mathfrak{R}(\nu)$; e) if for any atom $\mathfrak{A}$ of $\mu$ for which $U \in \mu(\mathfrak{F}(\mathfrak{A}))$ the filter $\mu(\mathfrak{F}(\mathfrak{U}))$ is convergent then the proper atoms of $\mu$ and $v$ coincide.

Let $W$ be a 0 -neighbourhood in $H$ such that if $H \neq\{0\}$ then $H-(W+W) \neq \varnothing$. By replacing $V$ with a smaller one we may assume $V-V \subset U$. We denote by $\Phi$ the set of atoms $\mathfrak{A}$ of $\mu$ such that for any $A \in \mathfrak{A}$ there exists $B \in \mathfrak{A}$ with $B \subset A$ and $\mu(B) \notin V$. By [1] Proposition $1.3 \mathrm{~d}$ ) any $\mathfrak{U} \in \Phi$ is a maximal element of $A$. Let $A \in \mathfrak{A}$, let

$$
\Phi(A):=\{\mathfrak{A} \in \Phi \mid A \in \mathfrak{A}\},
$$

and let $\mu_{A}$ be the restriction of $\mu$ to $\{B \in \Re \mid B \subset A\}$. Then $\mu_{A}$ is an exhaustive measure and for any $\mathfrak{A} \in \Phi(A)$ the set $\{B \in \mathfrak{A} \mid B \subset A\}$ is an atom of $\mu_{A}$. By Proposition 1.4 $\Phi(A)$ is finite. Hence for any infinite subset $\Psi$ of $\Phi$ we have $\cap_{\mathfrak{A} \in \Psi} \mathfrak{A}=\varnothing$. By [1] Proposition 1.6 there exists a family $\left(A_{\mathfrak{A}}^{\prime}\right)_{\mathfrak{Y} \in \Phi}$ in $\mathfrak{R}$ such that $A_{\mathfrak{Y}}^{\prime} \in \mathfrak{U} \backslash \mathfrak{U}^{\prime}$ for any $\mathfrak{A}, \mathfrak{U} \mathfrak{U}^{\prime} \in \Phi$, $\mathfrak{U} \neq \mathfrak{A}^{\prime}$. By [1] Proposition 2.5 for any $\mathfrak{U} \in \Phi$ and for any $A \in \mathfrak{U}$ there exists $K \in \mathfrak{R} \cap \mathfrak{U}$ with $K \subset A$.

Let $\mathfrak{A} \in \Phi$. By [1] Proposition $2.2 \mu(\mathfrak{F}(\mathfrak{U}))$ is a Cauchy filter. Hence there exists $A_{\mathfrak{A}}^{\prime \prime} \in \mathfrak{U}$ such that

$$
\mu\left(A_{\mathfrak{A}}^{\prime \prime} \backslash A\right) \in V
$$


for any $A \in \mathfrak{A}$ with $A \subset A_{\mathfrak{A}}^{\prime \prime}$. By [1] Proposition $1.3 \mathrm{c}$ ) and d)

$$
\mathfrak{R}_{0}:=\left\{A_{\mathfrak{A}}^{\prime \prime} \backslash A \mid A \in \mathfrak{U}\right\}
$$

is a $\sigma$-ring. The restriction of $\mu$ to $\Re_{0}$ is a measure such that $\mu\left(\Re_{0}\right) \subset V$. We deduce that the map

$$
A \mapsto u(\mu(A)): \Re_{0} \rightarrow H
$$

is a measure. Let us denote by $\widetilde{F}$ the filter on $\Re_{0}$ generated by the filter

$$
\left\{\left\{B \in \Re_{0} \mid A \subset B\right\} \mid A \in \Re_{0}\right\} .
$$

By Proposition 1.3 its image through the above map is a Cauchy filter. Hence there exists $A_{\mathfrak{Y}} \in \mathfrak{U}$ such that $A_{\mathfrak{U}} \subset A_{\mathfrak{U}}^{\prime} \cap A_{\mathfrak{U}}^{\prime \prime}$ and such that

$$
u\left(\mu\left(A_{\mathfrak{Q}}\right)-\mu(A)\right) \in W
$$

for any $A \in \mathfrak{A}$ with $A \subset A_{\mathfrak{U}}$.

Let $\left(x_{\mathfrak{U}}\right)_{\mathfrak{Q} \in \Phi}$ be an arbitrary family in $H$ (resp. in $H \backslash(W+W)$ if $H \neq\{0\})$. We set

$$
\begin{aligned}
& \mathfrak{R}^{\prime}:=\mathfrak{R} \backslash \cup \mathfrak{A}, \quad \mathfrak{K}^{\prime}:=\mathfrak{K} \backslash \cup \mathfrak{A} . \\
& \mathfrak{A} \in \Phi \quad \mathfrak{A} \in \Phi
\end{aligned}
$$

By Proposition 2.3 there exists a $\mathfrak{R}^{\prime}$-regular $H$-valued measure $\lambda$ on $\Re^{\prime}$ such that

for any $A \in \Re^{\prime}$ with

$$
\lambda(A)=u(\mu(A))
$$

$$
\left\{\mu(B) \mid B \in \Re^{\prime}, B \subset A\right\} \subset U .
$$

By Proposition 2.4 there exists a $\mathfrak{R}$-regular $H$-valued measure $v$ on $\Re$ equal to $\lambda$ on $\mathfrak{R}^{\prime}$ and equal to $x_{\mathfrak{U}}$ at $A_{\mathfrak{U}}$ for any $\mathfrak{U} \in \Phi$.

a) Let $A \in \Re$ with

$$
\{\mu(B) \mid B \in \Re, B \subset A\} \subset U .
$$

Then $A \in \mathfrak{R}^{\prime}$ and therefore

$$
v(A)=\lambda(A)=u(\mu(A)) .
$$

b) Let now $\mathfrak{A}$ be a proper atom of $v$. By a) $\mathfrak{N}(\mu) \subset \mathfrak{N}(v)$ and therefore $\mathfrak{A}$ is an atom of $\mu([1]$, Corollary 1.4). Assume that $\mu(\mathfrak{F}(\mathfrak{A}))$ converges to 0 . Let $A$ be a set of $\mathfrak{A}$ such that

$$
\{\mu(B) \mid B \in \mathfrak{H}, B \subset A\} \subset U \text {. }
$$

Since $u$ is an $\omega$-map it follows by a) that $v(\mathfrak{F}(\mathfrak{H}))$ converges to 0 and this is a contradiction. 
c) Let $\mathfrak{A} \in \Phi$, let $A \in \mathfrak{U}$ with $A \subset A_{\mathfrak{U}}$, and let $B \in \mathfrak{R}$ with $B \subset A_{\mathfrak{Q}} \backslash A$. Then $A \cup B \in \mathfrak{A}$ and $A \cup B \subset A_{\mathfrak{Q}}$ and therefore

$$
\begin{gathered}
\mu\left(A_{\mathfrak{A}}^{\prime \prime} \backslash A\right) \in V, \quad \mu\left(A_{\mathfrak{A}}^{\prime \prime} \backslash(A \cup B)\right) \in V, \\
\mu(B) \in V-V \subset U .
\end{gathered}
$$

We get by a)

$$
v\left(A_{\mathfrak{A}} \backslash A\right)=u\left(\mu\left(A_{\mathfrak{U}} \backslash A\right)\right)
$$

for any $A \in \mathfrak{A}$ with $A \subset A_{\mathfrak{Q}}$. Hence

$$
\begin{gathered}
x_{\mathfrak{A}}-v(A)=v\left(A_{\mathfrak{Q}}\right)-v(A)=u\left(\mu\left(A_{\mathfrak{Q}}\right)-\mu(A)\right) \in W, \\
v(A) \notin W
\end{gathered}
$$

for any $A \in \mathfrak{A}$ with $A \subset A_{\mathfrak{U}}$. It follows that $\mathfrak{A}$ is a proper atom of $v$.

Assume now that $u(x)=0$ implies $x=0$.

d) By a) $\mathfrak{R}(\mu) \subset \mathfrak{R}(v)$. Let $A \in \mathfrak{N}(v)$. Then $A \in \mathfrak{R}^{\prime}$ and therefore $A \in \mathfrak{N}(\lambda)$. By Proposition $2.3 A \in \mathfrak{N}(\mu)$.

e) Let $\mathfrak{U}$ be an atom of $\mu$ not belonging to $\Phi$. Then there exists $A \in \mathfrak{U}$ such that

$$
\{\mu(B) \mid B \in \mathfrak{A}, B \subset A\} \subset V .
$$

It follows $A \in \mathfrak{R}^{\prime}$. For any $B \in \mathfrak{A}$ with $B \subset A$ we get $A \backslash B \in \mathfrak{R}^{\prime}$,

$$
\begin{gathered}
\{\mu(C) \mid C \in \Re, C \subset A \backslash B\} \subset U, \\
v(A \backslash B)=\lambda(A \backslash B)=u(\mu(A \backslash B)), \\
v(B)=u(\mu(B)) .
\end{gathered}
$$

By the hypothesis $\mu(\mathfrak{F}(\mathfrak{U}))$ converges to an element $x \in U$. Since $u$ is an $\omega$-map $v(\mathfrak{F}(\mathfrak{U}))$ converges to $u(x)$. Since $u(x)=0$ implies $x=0$, $\mathfrak{A}$ is a proper atom of $\mu$ if and only if it is a proper atom of $\nu$.

\section{Control measures}

Throughout this section we shall denote by $\mathfrak{H}$ a class of Hausdorff topological commutative groups (resp. Hausdorff topological ordered commutative groups) closed with respect to the product operation and containing an $H \neq\{0\}$ and by $\mathbb{S s}$ the class of Hausdorff topological commutative groups $G$ such that for any $\mathfrak{R}$-regular G-valued measure $\mu$ on $\Re$ there exist an

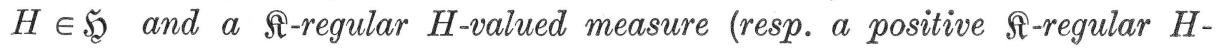
valued measure) $v$ on $\Re$ such that $\mathfrak{N}(\mu)=\mathfrak{N}(v)$. 
As variants we may require in addition some of the next conditions:

a) the proper atoms of $\mu$ and $\nu$ coincide;

b) $\mathfrak{R}(v)=\{A \in \Re \mid v(A)=0\}$;

c) if $\mu$ is exhaustive then $v$ is exhaustive.

By Theorem 2.5 any discrete topological commutative group belongs to $(5)$. If $\mu$ satisfies cce then a) is authomatically fulfilled ([1], Proposition $1.2 \mathrm{c}))$.

Proposition 3.1. The product of any family in Bs belongs to (BS .

Let $\left(G_{b}\right)_{t \in I}$ be a family in \&s and let $\mu$ be a $\Re$-regular $\Pi_{t \in I} G_{b}$-valued measure on $\Re$. For any $\iota \in I$ we denote by $u_{\iota}$ the projection $\Pi_{\lambda \in I} G_{\lambda} \rightarrow G_{\iota}$; then $u_{\iota} \circ \mu$ is a $\Re$-regular measure. Hence there exist for any $\iota \in I$ an $H_{\imath} \in \mathfrak{H}$ and a $\Re$-regular $H_{\imath}$-valued measure (resp. a positive $\Re$-regular $H_{\imath}$-valued measure) $v_{\imath}$ on $\Re$ such that

$$
\mathfrak{R}\left(\mu_{\iota}\right)=\mathfrak{N}\left(\nu_{\iota}\right) .
$$

We denote by $v$ the map

$$
A \mapsto\left(v_{\iota}(A)\right)_{\iota \in I}: \Re \rightarrow \prod_{\iota \in I} H_{\iota} .
$$

Then $v$ is a $\mathfrak{R}$-regular $\Pi_{\iota \in I} H_{\imath}$-valued measure (resp. a positive $\mathfrak{\AA}$-regular $\Pi_{\iota \in I} H_{\imath}$-valued measure) on $\Re$ such that

$$
\mathfrak{A}(\mu)=\mathfrak{N}(\nu) \text {. }
$$

If variant a) holds then we may choose the measures $v_{\iota}(\iota \in I)$ such that the proper atoms of $\mu_{t}$ and $\nu_{\iota}$ coincide. We deduce that the proper atoms of $\mu$ and $v$ coincide.

If variant $b$ ) holds then.

$$
\Re\left(\nu_{\iota}\right)=\left\{A \in \Re \mid \nu_{\iota}(A)=0\right\}
$$

for any $\iota \in I$ and therefore

$$
\Re(v)=\{A \in \Re \mid v(A)=0\} .
$$

If variant c) holds and if $\mu$ is exhaustive then for any $\iota \in I, \quad v_{\iota}$ is exhaustive and therefore $v$ is exhaustive.

Remark. Since any subgroup of a group of $\mathbb{S S}$ belongs to (4S it follows that the projective limits of groups of (5) belong to 15 .

Th e o r e m 3.2. Let $G$ be a Hausdorff topological commutative group and let $U$ be a 0-neighbourhood in $G$ such that for any $a \in U \backslash\{0\}$ there exist a continuous semi-value $p_{a}$ on $G$ with $p_{a}(a) \leqq 1$ and an $\omega$-map $u_{a}$ of

$$
U_{a}:=\left\{x \in G \mid p_{a}(x) \leqq 1\right\}
$$

into a group of (S) not vanishing at $a$. Then $G \in \mathbb{S}$. 
We may assume $G$ non-discrete. Let $\mu$ be a $\mathfrak{R}$-regular $G$-valued measure on $\mathfrak{R}$. Let us denote by $\mathfrak{B}$ the set of 0 -neighbourhoods in $G$ and by $U^{\prime}$ the set $U \backslash\{0\}$. By Theorem 2.5 there exist for any $(a, V) \in U^{\prime} \times \mathfrak{S}$ an $G_{a, V} \in \mathbb{B S}$ and a $\Re$-regular $G_{a, V}$-valued measure $\lambda_{a, V}$ on $\Re$ such that: a) $\lambda_{a, V}(A)=u_{a}(\mu(A))$ for any $A \in \Re$ with

$$
\{\mu(B) \mid B \in \Re, B \subset A\} \subset U_{a} ;
$$

b) any proper atom of $\lambda_{a, V}$ is a proper atom of $\mu$; c) if $\mathfrak{A}$ is an atom of $\mu$ such that for any $A \in \mathfrak{A}$ there exists $B \in \mathfrak{A}$ with $B \subset A$ and $\mu(B) \notin V$ then $\mathfrak{A}$ is a proper atom of $\lambda_{a, V}$. We denote by $\lambda$ the map

$$
A \mapsto\left(\lambda_{a, V}(A)\right)_{(a, V) \in U^{\prime} \times \mathfrak{B}}: \mathfrak{R} \rightarrow \underset{(a, V) \in U^{\prime} \times \mathfrak{Z}}{\Pi} G_{a, V} .
$$

Then $\lambda$ is a $\mathfrak{\Re}$-regular $\Pi_{(a, V) \in U^{\prime} \times \mathfrak{B}} G_{a, V}$-valued measure on $\Re$. By a) we have $\mathfrak{N}(\mu) \subset \mathfrak{N}(\lambda)$.

Let $\mathfrak{A}$ be a proper atom of $\lambda$. Then there exists $(a, V) \in U^{\prime} \times \mathfrak{B}$ such that $\lambda_{a, V}(\mathfrak{F}(\mathfrak{A}))$ does not converge to 0 . Since $\mathfrak{R}(\lambda) \subset \mathfrak{R}\left(\lambda_{a, V}\right)$, $\mathfrak{A}$ is a proper atom of $\lambda_{a, V}([1]$, Corollary 1.4$)$. By b) $\mathfrak{U}$ is a proper atom of $\mu$.

Let $\mathfrak{A}$ be a proper atom of $\mu$. Then there exists $V \in \mathfrak{Z}$ such that for any $A \in \mathfrak{A}$ there exists $B \in \mathfrak{A}$ with $B \subset A$ and $\mu(B) \notin V$. By c) $\mathfrak{A}$ is a proper atom of $\lambda_{a, V}$ for any $a \in U^{\prime}$.

Let $A \in \mathfrak{R} \backslash \mathfrak{R}(\mu)$ and let $\mathfrak{A}$ be a proper atom of $\mu$ containing $A$. By the above considerations there exists $V \in \mathfrak{B}$ such that $\mathfrak{A}$ is a proper atom of $\lambda_{a, V}$ for any $a \in U^{\prime}$. Since $G$ is not discrete we get $A \notin \Re(\lambda)$.

Let $A \in \Re \backslash \mathfrak{R}(\mu)$ such that no proper atom of $\mu$ contains $A$. By Proposition 1.2 there exists $B \in \mathfrak{R} \backslash \mathfrak{N}(\mu)$ contained in $A$ such that $\mu(B) \in U^{\prime}$. Then $\mu(B) \in U_{\mu(B)}$ and

$$
u_{\mu(B)}(\mu(B)) \neq 0 \text {. }
$$

By Proposition 2.3 we get $B \notin \Re\left(\lambda_{\mu(B), V}\right)$ for any $V \in \mathfrak{V}$ and therefore $A \notin \mathfrak{N}(\lambda)$. Hence $\mathfrak{N}(\mu)=\mathfrak{N}(\lambda)$.

Let $\mathfrak{A}$ be a proper atom of $\mu$. Then $A$ is an atom of $\lambda$ ([1], Corollary 1.4) and by the above considerations it is even a proper atom of $\lambda$.

Assume that $\mu$ is exhaustive. Let $(a, V) \in U^{\prime} \times \mathfrak{S}$ and let $\left(A_{n}\right)_{n \in N}$ be a disjoint sequence in $\Re$. By Proposition 1.6 there exists $m \in N$ such that

$$
\left\{\mu(A) \mid A \in \Re, A \subset A_{n}\right\} \subset U_{a}
$$

for any $n \geqq m$. We get by a)

$$
\lambda_{a, V}\left(A_{n}\right)=u_{a}\left(\mu\left(A_{n}\right)\right)
$$


for any $n \geqq m$. Since $\left(\mu\left(A_{n}\right)\right)_{n \in N}$ converges to 0 and since $u_{a}$ is an $\omega$-map it follows that $\left(\lambda_{a, V}\left(A_{n}\right)\right)_{n \in \boldsymbol{N}}$ converges to 0 . Hence $\left(\lambda\left(A_{n}\right)\right)_{n \in \boldsymbol{N}}$ converges to 0 and $\lambda$ is exhaustive.

By Proposition $3.1 \Pi_{(a, V) \in U^{\prime} \times \mathfrak{S}} G_{a, V} \in \mathbb{S}$. Hence there exist an $H \in \mathfrak{H}$ and a $\mathfrak{\AA}$-regular $H$-valued measure (resp. a positive $\mathfrak{R}$-regular $H$-valued measure) $v$ on $\mathfrak{R}$ such that $\mathfrak{l}(\lambda)=\mathfrak{N}(\nu)$. It follows that $\mathfrak{N}(\mu)=\mathfrak{N}(\nu)$.

If variant a) holds we may require in addition that the proper atoms of $\lambda$ and $\nu$ coincide. Then the proper atoms of $\mu$ and $\nu$ coincide.

If variant b) holds we may require

$$
\mathfrak{R}(\nu)=\{A \in \mathfrak{R} \mid \nu(A)=0\} .
$$

If variant c) holds and if $\mu$ is exhaustive, then by the above considerations $\lambda$ is exhaustive and therefore we may choose an exhaustive $v$.

Corollary 3.3. Let $G$ be a Hausdorff topological commutative group and let $U$ be a 0-neighbourhood in $G$ such that for any $a \in U \backslash\{0\}$ there exist a continuous semi-value $p_{a}$ on $G$ with $p_{a}(a) \leqq 1$ and an $\omega$-map $u_{a}$ of

$$
U_{a}:=\left\{x \in G \mid p_{a}(x) \leqq 1\right\}
$$

into $R$ not vanishing at $a$. Then for any $\mathfrak{\AA}$-regular exhaustive $G$-valued measure $\mu$ on $\Re$ satisfying ccc there exists a positive $\mathfrak{A}$-regular exhaustive real valued measure $v$ on $\Re$ such that $\mathfrak{N}(\mu)=\mathfrak{R}(v)$.

By the theorem there exist a set $X$ and an exhaustive $\Re$-regular $\boldsymbol{R}^{X}$-valued measure $\lambda$ on $\Re$ such that $\mathfrak{N}(\mu)=\mathfrak{N}(\lambda)$. Let us denote for any $x \in X$ by $\pi_{x}$ the corresponding projection $\boldsymbol{R}^{X} \rightarrow \boldsymbol{R}$. By [3] Theorem 1 there exists a sequence $\left(x_{n}\right)_{n \in \boldsymbol{N}}$ in $X$ such that

$$
\mathfrak{N}(\lambda)=\bigcap_{n \in \boldsymbol{N}} \mathfrak{N}\left(\pi_{x_{n}} \circ \mu\right) .
$$

By Proposition 1.6

$$
\alpha_{x}:=\sup _{A \in \Re}\left|\pi_{x} \circ \lambda\right|(A)<\infty
$$

for any $x \in X$. The measure

$$
v:=\sum_{n \in N} \frac{1}{2^{n} \alpha_{x_{n}}}\left|\pi_{x_{n}} \circ \lambda\right|
$$

possesses the required properties.

From this corollary we may deduce the following result of K. Musiał ([2], Theorem 8):

Corollary 3.4. Let $\mu$ be a $\mathfrak{R}$-regular exhaustive measure on $\Re$ satisfying ccc with values in a locally compact commutative group. Then 
there exists a positive $\mathfrak{\AA}$-regular exhaustive real valued measure $v$ on $\Re$ such that $\mathfrak{R}(\mu)=\mathfrak{N}(\nu)$.

\section{References}

[1] Constantinescu, C.: Atoms of group valued measures. - Comment. Math. Helv. (to appear).

[2] Musiat, K.: Absolute continuity and the range of group valued measure. . Bull. Acad. Polon. Sci. Sér. Sci. Math. Astronom. Phys. 21, 1973, 105-113.

[3] -》- Absolute continuity of vector measures. - Colloq. Math. 27, 1973, $319-$ 321 .

Technische Universität Hannover

Institut für Mathematik

Welfengarten 1

D 3 Hannover

Bundesrepublik Deutschland

Received 7 July 1975 